\title{
Crecimiento después de desnutrición grave precoz
}

\author{
Jorge Alvear A. ${ }^{1}$; Margarita Vial R. ${ }^{1}$; Carmen Artaza B. ${ }^{1}$;
}

Growth after early, severe, undenutrition

\begin{abstract}
In order to investigate growth foliowing early severe mainutrition, two groups of 40 children and their mothers were studied, one of patients recovered from early protein energy malnutrition (PEM) at a closed center for nutritional recoverv and another one of children under the seme socioeconomic condition but who had never been malnourished. Both groups were matched for sex and age, and were followed by the same professional team for 9 years. A significant difference in mother's size (154.8 \pm 5.2 vs. $150 \pm 3.6 \mathrm{~cm}$ ) and in patients size at birth (males $3,453.5 \pm 522.3$ vs $3,018.2 \pm 490.3$ and females $3,328.4 \pm 563.4$ vs. 2,654.6 $\pm 579.6 \mathrm{~g}$ ) was found in favour of the control group $(p<0.01)$. There were significant differences in height for age $(\mathrm{H} / \mathrm{A} /$ and weight for age (W/A) ( $p<0.001$ ) but not in weight for height ratios between children for both groups (fig. 1 and 2 ). No differences were detected in bone age. Growth velocity was similar in both groups of boys $(10.40 \pm 2.3 \mathrm{vs} .981 \pm 1.40 \mathrm{~cm}$ and $12.94 \pm 2.53$ vs. $12.80 \pm 1.64 \mathrm{~cm}$ from 5 to 7 and 7 to 9 years of age respectively) but it was significantly greater in controf girls $\{11.22 \pm 2.70$ vs, $9.30 \pm 1.60$ and $15.60 \pm 1.66$ vs. $12.80 \pm 2.20 \mathrm{~cm} 5$ to 7 and 7 to 9 years respectively, $p<0.01\}$. These findings show that malnutrition before 2 vears of age may produce long term effects on growth in children of low socioeconomic condition specially if, after treatment, they are turned back to the same unfavourabie environment.

(Key words: protein-calorie malrutrition, growth, child development follow up studies.)
\end{abstract}

El crecimiento humano es el resultado de la interacción de factores genéticos $y$ ambientales que se influencian entre sí de manera recíproca y continua.

La informaciôn genética determinaría la calidad y cantidad de crecimiento. Esta depende de la frecuencia y velocidad de la djvisión celular, de la sensibilidad de los tejidos a estŕmulos de crecimiento, de la edad de pubertad y el cierre de los cartilagos de crecimiento. Sin embar. go, el crecimiento es influenciado también por el medio ambiente, La nutrición, los estímulos biológicos y patológicos, el clima y las infecciones intercurrentes son los lamados factores ambientales o extrínsecos de crecimiento. Considerando estos antecedentes, la genética explicaría la mayor parte de los retardos de crecimiento en los países desarrollados y serían los factores ambientales los que determinan los retrasos en los países en vía de desarrollo ${ }^{1-4}$.

El retardo de crecimiento provocado por la desnutrición puede afectar hasta $50 \%$ de los

1. INTA. Instituto de Nutrición y Tecnología de los Alimentos, Universidad de Chile. niños menores de 5 años en paises en vías de desarrollo. En muchos casos el problema comienza "in útero" ya que $20 \%$ de los niños del llamado tercer mundo nacen con un peso menor de $2500 \mathrm{~g}^{5,6}$.

Se ha hecho notar en forma especial la importancia de la madre sobre el niño en crecimiento, considerando de modo muy especial la influencia biológica que ejerce el tamaño materno sobre peso fetal y su influencia sobre crecimiento postnatal y desarrollo de personalidad infan. til $^{7,8}$. En estudios del crecimiento humano se han observado diferencias étnicas en proporciones corporales como el largo de extremidades inferiores (o sea, la diferencia entre talla total y talla sentada) y esto podría estar influenciado por factores étnicos y geográficos. El largo del tronco y cabeza serían más resistentes que el segmento inferior a variaciones del ambiente, por lo que predicciones de talla sentada resultan independientes del nivel socioeconómico ${ }^{7-9}$.

Se ha observado que la desnutrición produce retardo de la maduración ósea. Este hecho se considera muy importante porque si la desnutrición retrasa la maduración de los cartílagos de crecimiento esto prolongaría el período del 
crecimiento longitudinal permitiendo crecimiento recuperacional si la situación nutricional mejorara ${ }^{9-12}$.

\section{Material y Método}

Se estudiaron en forma longitudinal 40 niños de extrema pobreza que requirieton tratamiento nutricional antes de $\operatorname{los} 2$ años de edad en un centro cerra. do de recuperación nutricional (CONIN). Estos niños fueron derivados de hs polictínicas del área suroriente de Santiago con desnutrición marásmica grave, grado UI, descompensada. Ingresaron con una relación de peso esperado para la edad de $58,5 \pm 8,8 \%$, en tanto que la relacjón peso para la talk era de $83,5 \pm 8,1 \%$. La tecuperación integral de los hactantes se lopró en $133,2 \pm 23$ dias, tiempo en que alcanzaron porcentajes de peso para la dad de $83,9 \%$ y relación peso para la talla de $103 \pm 9,5 \%$ según estándares OMS.

Estos niños se oompararon con $\mathbf{4 0}$ niños del mismo nivel socioeconómioo sin el antecedente de desnutrición, que asistian a los mismos jardines infantiles y galas cunas que los del grupo de estudio, elegidos al azar con el consentimiento escrito de sus padres. En ambos grupos se registraron pesos y tallas de racimiento, obtenidos de las maternidades donde nacjeron, y se parearon según edad y sexo. Pacientes y controles fueron controlados mensualmente por 6 meses después del alta y 2 veces al año por 9 años por el mismo equipo profesional (médi$\infty$, asistente social, enfermera). Durante los controles se les practicó examen de salud, se trataron las enfermedades intercurrentes, se registraron sus mediciones a ntropométricas $y$ se realizó entrevista social. El peso se determinó desnudo hasta $\operatorname{los} 10 \mathrm{~g}$ más próximos. Talla de pie con talones, nalgas y cabeza apoyando firmemente en el antropómetro. Las circunferencias de brazo y cabeza se tomaros usando huincha de fibra de vidrio inextensibie. La circunferencia de cráneo se tomó considerando los puntos más prominentes de huesos frontales y occipucio hasta el $0,1 \mathrm{~mm}$ próximo. La circunferencia de brazo, tomando el tercio medio de brazo izquierdo, Los pliegues cutáneos se midieron con calibrador Harpender usando una presión de $10 \mathrm{~g} \mathrm{x} \mathrm{mm^{2 }}$ hasta el $0,1 \mathrm{~mm}$ más próximo. Se realizaron 3 mediciones, obteniéndose el valor promedio en hemicuerpo izquiexdo de acuerdo al programa biológico internacional ${ }^{13}$. Las mediciones antropométricas fueron realizadas por dos investigadores en forma independiente, existiendo coincidencia en los valores consignados con variaciones de $0,5 \mathrm{~cm}$ en talla y $10 \mathrm{~g}$ en peso.

El estado nutricional se evaluó en términos de por. centaje de peso para edad, talta para edad, peso para talla y circunferencia de cráneo para edad, de acuerdo a estándares OMS.

Area grasa $y$ area magra se determinaron usando h relación circunferencia de brazo y pliegue cutáneo según estándares Frisancho ${ }^{14}$.

El análisis estadístico fue realizado mediante un computador IBM, aplicando diferentes pruebas estadísticas como $t$ de Student, chi cuadrado, coeficiente de correlación simple y múltiple, dependiendo del nivel de medición y de hs relaciones a establecer entre las yariables.
En un subgrupo de 25 niños a los 5, 7 y 9 años de seguimiento se practicó $\mathbf{R x}$ de carpo para estudiar edad ósea y se practicó antropometría a sus madres biológicas. La edad ósea se obtuvo en una radiografía de carpo de mano izquierda usando el atlas de GreulichPyle como referencia. Las radiografías fueron analizadas en doble ciego por dos observadores, existiendo coincidencia en las observaciones ${ }^{15}$.

\section{Resultados}

La antropometría de los nir̃os estudiados está resumida en las figuras 1 y 2 donde se describe la evolución del peso para la edad y peso para la talla según estándares OMS. Se encontraron diferencias entre el grupo de estudio con antecedentes de desnutrición calórico proteica (DCP) grave tratada y el grupo control, en los 3 cortes del seguimiento. El grupo control, a pesar de sus desfavorables condiciones ambjentales, evolucionó en el percentil 50 de peso y talla, a diferencia del grupo con antecedentes de desnutrición, que lo hizo en percentil 3, tanto en hombres como mujeres. Cuando se analizan los niños según peso para la talla estas diferencias desaparecen independiente del sexo.

La velocidad de crecimiento en varones expresada en centímetros ganados a los 5 y 7 años de edad con (n: 8) y sin (n: 10) antecedentes de DCP $(x \pm D E)$ no mostró diferencias significativas $(9,80 \pm 1,40$ versus $10,40 \pm 2,30)$. En el periodo 7 a 9 años se observó igual comportamiento $(12,80 \pm 1,64$ versus $12,94 \pm 2,53)$. Sin embargo, en las mujeres se observaron dife. rencias significativas en el período de 5 a 7 años entre el grupo con antecedentes DCP ( $n: 8): 9,30 \pm 1,60$ versus sin antecedentes (n: 10), 11,22 $\pm 2,70$ (p $<0,01)$, y en el perío. do. 7 a 9 años ocurrió algo similar con 12,80 $\pm 2,20$ versus $15,60 \pm 1,66$. El dimorfismo sexual observado en el grupo control no apareció en el grupo con antecedentes de DCP en el período 7 a 9 años: varones (n: 9), 12,94 \pm $2,53$ versus mujeres ( $n: 10), 15,60 \pm 1,66 . \mathrm{El}$ análisis del estado nutricional medido por la evolución del área magra y grasa no mostró diferencias significativas entre los grupos $y$ separados por sexo.

El estudio de la evolución de las edades óseas analizadas según Atlas de Greulich y Pyle mostró retraso de las edades óseas de toda la muestra estudiada, independiente de su historia y evaluación del estado nutricional al momento de la radiografía de carpo. No se encontraron dife- 


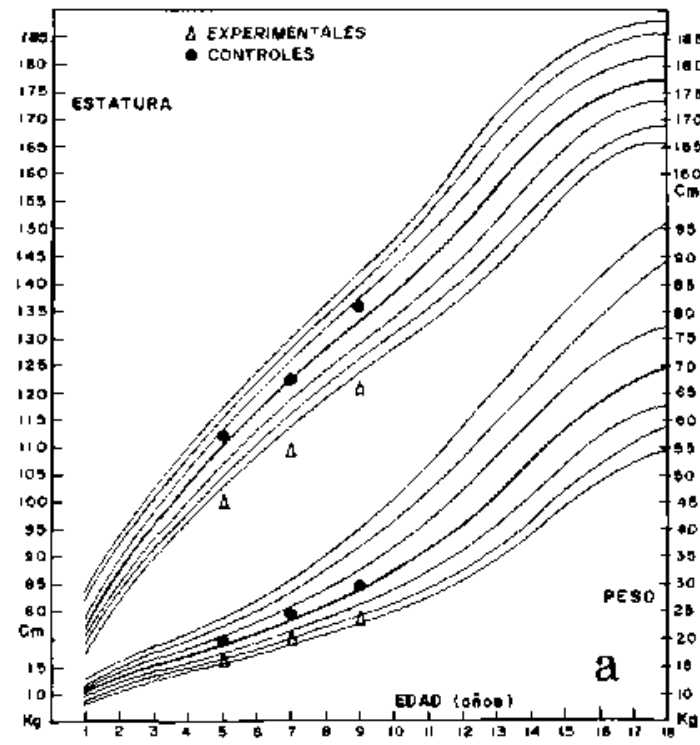

Figura 1: Evolución antropamétrica de las relaciones peso/edad y talla (estatura)/edad (promedios) (OMS) para niños (a) y niñas (b) con y sin antecedentes de

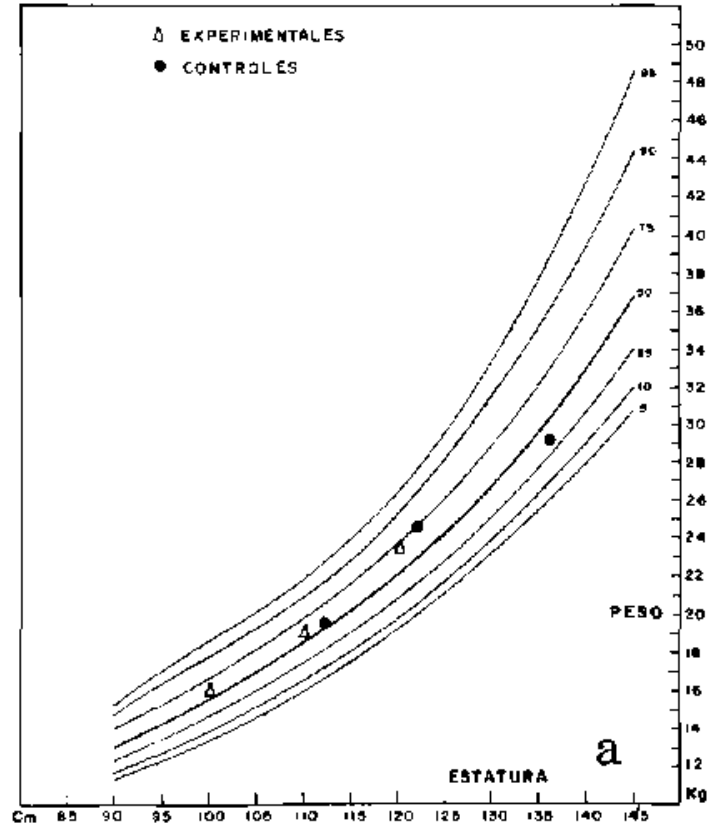

Figura 2: Evolución peso para ba talla (OMS) para niños (a) y niñas (b) con y sin antecedentes de desnutri-

rencias sigrificativas entre los grupos estudiados y separados por sexo. Las diferencias de las edades óseas entre los sexos (dimorfismo sexual) a los 5 años de edad desaparecieron a los 7 años como consecuencia de sobreaceleración de la

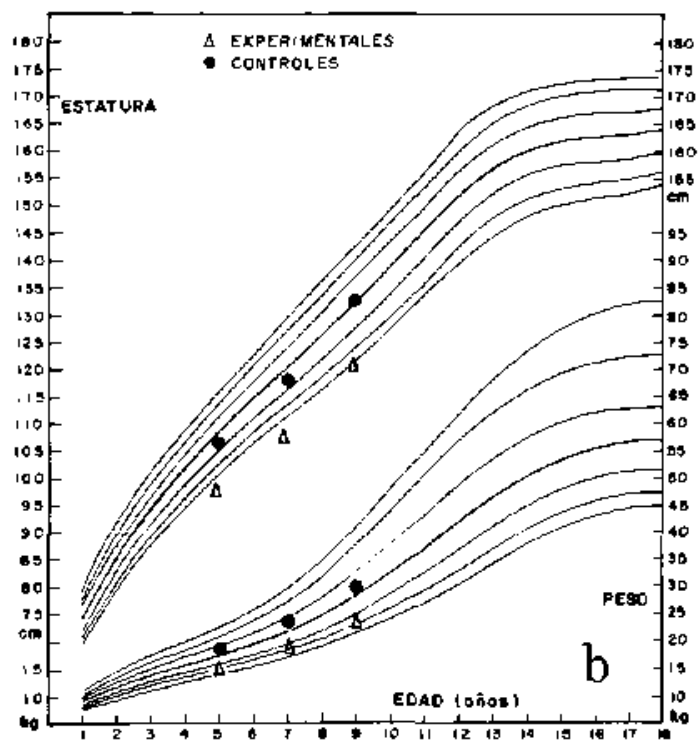

desnutrición calórica proteica grave precoz a los 5, 7 y 9 años de edad.

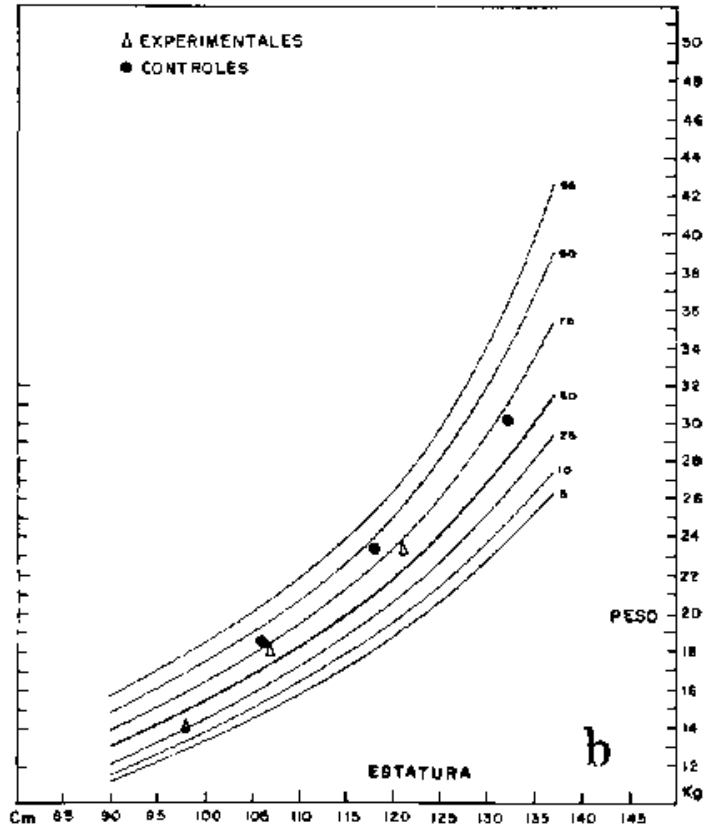

ción calórica proteica grave precoz a los 5,7 y 9 años de edad.

maduración ósea en los hombres, independiente de su historia nutricional (tabla 1).

El análisis de los antecedentes perinatales con especial interés en la antropometría de nacimiento permitió una clara diferenciación de los grupos 
Tabla 1

Edades óseas (\% Greulich y Pyle) en niños con y sin antecedentes DCP precoz a $\operatorname{los} 5,7$ y 9 años de vida

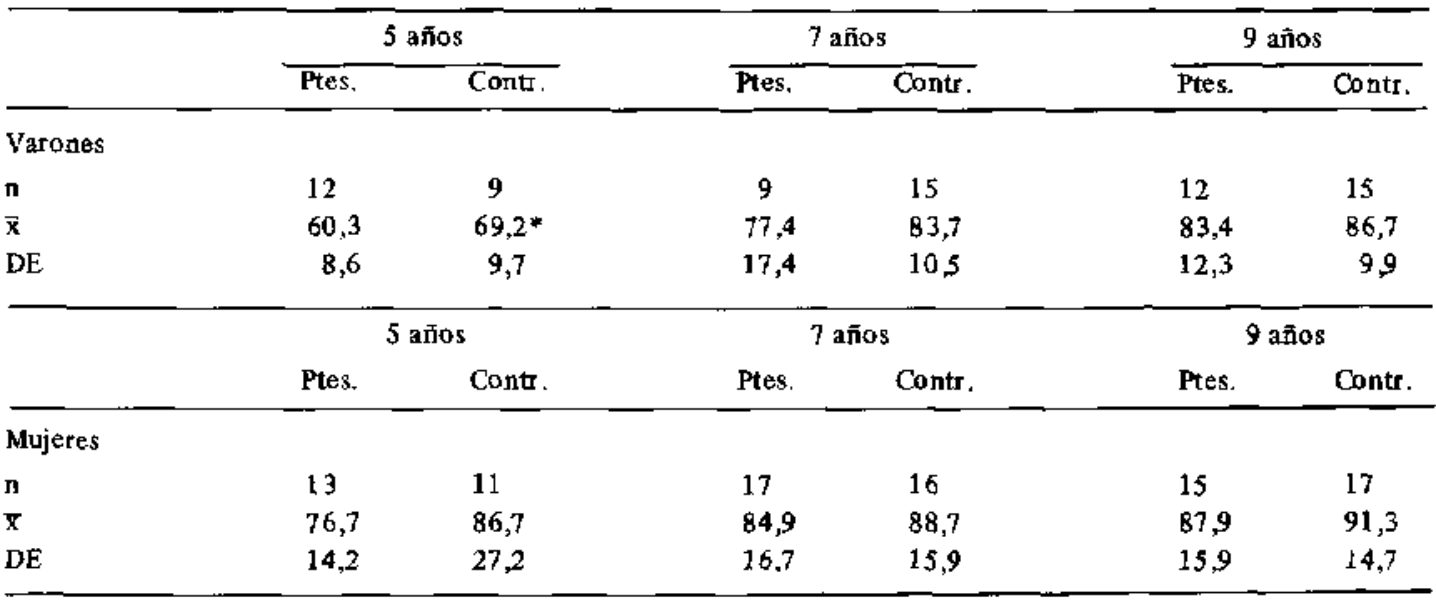

Ptes,; niños con antecedentes de desnutrición.

Contr: Controles sanos.

*: $\mathrm{p}<0,01$.

( $\mathrm{p}<0,001$ ), tanto para peso como para talla de ficativas, de casi $5 \mathrm{~cm}$, entre las tallas maternas nacimiento, que favorecieron al grupo control, en favor del grupo control: $150,0 \pm 3,6$ versus pues estos ninos nacieron con 400 a $600 \mathrm{~g} \mathrm{y} 3 \mathrm{~cm} \quad 154,8 \pm 5,2 \mathrm{~cm}(\mathrm{p}<0,01)$. La circunferencia de más de peso y talla que los que requirieron tra- cráneo seguia la misma modalidad que la talla tamiento de desnutrición (tabla 2).

El estado nutricional de 24 madres de niños con antecedentes DCP, comparadas con $35 \mathrm{ma}$. dres del grupo control, mostró diferencias signi$(\mathrm{p}<0,01)$, sin diferencias entre los pesos de ambos grupos, ni entre la relacjón de segmentos superior ni inferior.

Tabla 2

Esta do nutricional al nacer de los nifios con antecedentes DCP $y$ controles

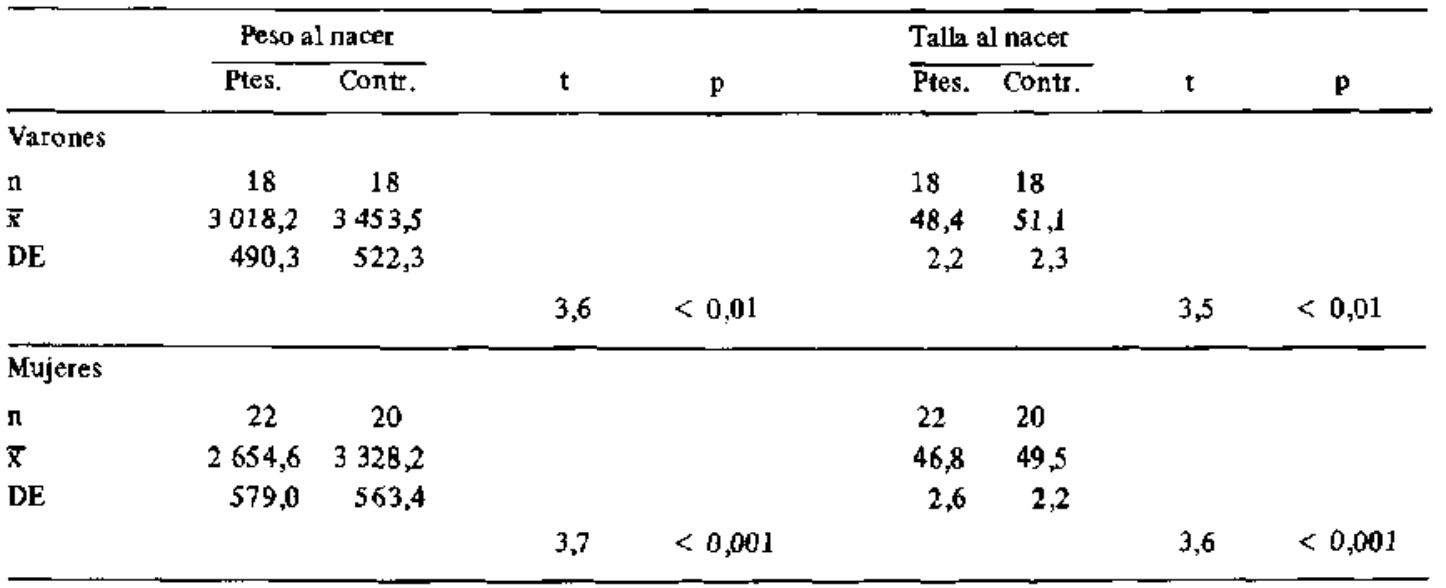

Ptes.: Niños con antecedentes de desnutrición.

Contr,: Controles sanos. 


\section{Discusión}

La desnutrición en la infancia es aún un pro. blema muy importante en los países del tercer mundo, tanto por su asociación con niveles altos de morbimortalidad precoz como por las secuelas a largo plazo que se observan ${ }^{16}$. La experien. cia que mostramos proviene de un programa de rehabilitaciốn integral de niños desnutridos graves, de los que probablemente habrian muerto de no haber mediado este programa; sin embargo. observamos que a pesar de la intervención nutricional el hecho de volver al medio de origen repercute en forma negativa sobre su crecimiento.

Es importante recordar que el largo del cuerpo está determinado por la longitud del esqueleto y que los cambios en la dieta se demoran más en traducirse en cambios de su ritmo de crecimiento y que una vez que éste se altera también tarda más en retornar el ritmo adecuado.

Nuestro estudio muestra que la desnutrición comenzaría antes del nacimiento, considerando los pesos de nacimiento del grupo que requirió tratamiento nutricional, $y$ el análisis de las tallas maternas sugeriría que el tamaño que alcanza el feto in útero dependeria en cierta forma del tamario de la madre, observación que está de acuerdo con antecedentes publicados en la literatura. Sir embargo, existe información que relaciona el peso de nacimiento con la dieta matema ${ }^{17-19}$. En nuestro estudio probablemente juegan estos dos factores en forma que no podemos determinar claramente (tamaño materno y dieta insuficiente durante el embarazo). E1 hecho de que madres del grupo de estudio y control no sean diferentes en relación a segmentos nos demuestra factores genéticos o nutricjonales de larga data. Si las madres del grupo de estudio hubiesen sufrido desnutrición exclusivamente, su segmento inferior debería haber estado comprometido.

Cuando analizamos la evolución del ritmo de crecimiento vemos que la desnutrición afecta la velocidad en que se ganan centímetros en el tiempo y que los distintos segmentos crecen en forma diferente, según el antecedente nutricional, desapareciendo o retrasando el dimorfismo sexual que aparece en el grupo control, situación que podrá objetivarse en otro corte por tener este estudio el carácter de longitudinal.

Llama la atención el arálisis de las edades óseas de ambos grupos que se comportan en for- tha similar a lo largo del tiempo. Parece importante destacar el aspecto genético, objetivado con el hecho de que ambos grupos presentan retardo en la maduración ósea respecto a los patrones americanos de Greulich y Pyle, por lo que es indispensable estimular la investigación de patrones nacionales.

\section{Resumen}

Con el propósito de investigar el crecimiento en niños que han suftido desnutrición calórica proteica severa precozmente se comparó la evolución antropométrica en 40 de tales nijos, previamente tratados y recuperados en un centro de recuperación nutricional, y la estatura de sus madres, con las de 40 controles sanos de las mismas edades, sexos y condición socioeconómica, a lo largo de 9 años y por el mismo observador. Se encontraron diferencias significativas en favor de los controles en la talla de las madres (154,8 \pm 5,2 vs. $150 \pm 3,6 \mathrm{~cm}$ ) y en el peso de nacimiento de los niños (varones $3453,5 \pm 522,3$ vs. $3018,2 \pm 490,3$ y mujeres $3328,4 \pm 563,4$ vs. $2654,6 \pm 579,6 \mathrm{~g}, \mathrm{p}<0,01)$. También había diferencias significativas en las relaciones talia/ edad y peso/edad ( $p<0,001)$, pero no en la de peso/talla entre ambos grupos. No se detectaron diferencias en ta edad ósea. La velocidad de crecimiento fue semejante en los varones de ambas muestras $(10,4 \pm 2,3$ vs. $9,81 \pm 1,4 \mathrm{~cm}$ y $12,94 \pm$ 2,53 vs, $12,8 \pm 1,64$ cm de 5 a 7 y de 7 a 9 años respectivamente), pero fue significativamente no. yor en las niñas controles $(11,22 \pm 2,7$ vs, $9,3 \pm$ 1,6 y $15,6 \pm 1,66$ vs. $12,8 \pm 2,2 \mathrm{~cm}$ de 5 a 7 y 7 a 9 años respectivamente, $p<0,01$ ). Estos hallazgos sugieren que la desnutrición calórica proteica antes de la edad de 2 años puede producir efectos en el largo plazo en niños de baja condición socioeconómica que son devueltos al medioambiente desfavorable después de tratarlos y recuperarlos de la desnutrición.

(Palabras claves: desnutrición calórica pro. teica, crecimiento, seguimiento.)

\section{Referencias}

1. Susanne, C.: Genetic and environmental influences on morphological characteristics. Ann Hum Biol 1975; 2: 279-287. 
2. Bogin, B.A.; Mac Veon, R, B.: Growth in height and weight of urban Guatemala primary school children of low and high socioeconomic class. Hum Biol 1978; $50: 477-487$.

3. Daman, A.: Stature increase among Italian Americans Environmental, genetic or both. Am J Phys Anthropol 1965;23; 401-408.

4. Wales, J.K.H.; Mitner, R.D.G.: Knemometry in assessment of linear growth. Arch Dis Child 1987 ; 62: $166-171$.

5. Habicht, J.P.; Martorel, R.: Yarbrough, C.; Malina. $R . ;$ Klein, $R . E_{.:}$Height and weight standards for preschool children how relevant are ethnic differences in growth potentibl. Lancet 1974; 1: $611 \cdot 615$.

6. Bengoa, J.M.: The problem of malnutrition WHO Chron 1974; $38: 3$.

7. Rosso. P.: Nutrition and matemal fetal exchange. Am J Clin Nutr 1981 ; $34: 744$.

8. Widdowson, E.M.: 1955, Reproduction and obesity. Voeding, 16-94.

9. McKeown, T.; Record, R.G.: Influence of ptenatal enviroment on corretation between birth weight and parenteral height. Am J Hum Genet 1954; 6457.

10. McCance, R.A.: Food growth and time. Lancet $1962 ; 2: 621-671$.

11. Alvear, J.; Artuza, C.; Vial, M.; Guerrero, S.; Muzzo, S. Physical growth and bone age of survivors of protein energy malnutrition. Arch Dis Child 1986; 61: 257-262.
12. Alcázar, M.L.; Alvear, J.; Muzzo, S.: Influencia de la nutricjón en el desarrollo óseo. Arch Latinoam Nut 1984; 34: 298-307.

13. Weiner, J.S.: lourie, $f$. A.: Human Biology, a guide to fields methods. International Biological Programme. Oxford: Blackwell Scientific Publications, 1969.

14. Frisancho, R.: New norms of upper limb fat and muscle areas for assessment of nutritional status. Am J Clin Nutr 1981; 34: 2540-2545.

15. Grewlich, W.E.; Pyle, S.I.: Radiografic atlas of skeletal development of the hand and wrist, 2nd ed. London: Stanford Press, 1959:p. 256.

16. McCormick, M.C.: The contribution of low birth weight to infant mortality and childhood morbidity. N Engl J Med $1985 ; 312: 82-90$.

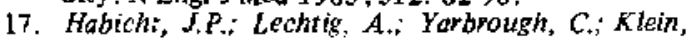
$R . E_{v}$ Maternal nutrition, birth weight and infant mortality. In: Ciba Foundation. Size at birth. Amsterdam; Elsevier/Excerpta Medica, 1974, pp. $353-377$.

18. Lechtig, A.; Delgodo, $\boldsymbol{H}$, ; Lasky, R,E, et al.: Maternal nutrition and fetal growth in developing societies: socioeconomic factors. Am J Dis Child $1975 ; 129: 434-437$.

19. Viegas, O.A.C., Scott, P.H., Cole, T.J. et al.: Dietary proteinenergy supplementation of pregnant asian mothers at Sorrento Birmingham I: unselective during second and third trimester. II: selective during seoond and third trimester only. $\mathrm{Br}$ Med J1982;285: 589-595. 